\title{
Bridge Damage Identification by Ground-based Synthetic Aperture Radar Using Blind Source Separation and Noise Reduction Technology
}

\author{
Qian-Hao Cheng, Qiang Chen, Hui Wang, and Xiang-Lei Liu* \\ School of Geomatics and Urban Spatial Information, Beijing University of Civil Engineering and Architecture, \\ Huangcun Town, Daxing District, Beijing 102616, China
}

(Received July 22, 2020; accepted November 27, 2020)

Keywords: damage identification of bridges, blind source separation, fast Fourier transform, groundbased synthetic aperture radar, second-order blind identification

Ground-based synthetic aperture radar (GBSAR) is regarded as an important monitoring technique for bridge damage identification. However, the interference effect of noise signals on bridge damage identification reduces its effectiveness. In this study, we proposed a blind source separation (BSS) technology based on a second-order blind identification (SOBI) algorithm, which was applied to bridge damage identification with GBSAR. We used two groups of simulated experiments with different frequencies to verify the feasibility of this method. Then, we conducted an experiment using actual GBSAR data for the bridge. To verify the effectiveness of the algorithm, we compared the frequencies of the bridge signals that were identified by BSS and fast Fourier transform (FFT). The results showed that the frequency of the damaged bridge was $7.324 \%$ higher than that of the healthy bridge, and the frequency increase of the monitoring signal processed by BSS was clearly accurate. The frequency of the damaged bridge was $59.819 \%$ higher than that of the healthy bridge. These findings showed that the SOBI method can be used to separate signals in order to obtain the signal source of the damage more efficiently, reduce the interference of other signal sources, and significantly improve the effectiveness of bridge damage identification.

\section{Introduction}

Urban bridges are essential for transportation in cities, but long-term vehicle loads, snow and rain, and other natural phenomena pose a threat to urban bridges, ${ }^{(1)}$ resulting in small deformations of the bridges. If timely protection measures are not taken, bridges may eventually collapse. As an important part of bridge health monitoring systems, bridge damage identification $^{(2)}$ plays an important role in the prediction of bridge damage. This technology uses relevant instruments to monitor the situation of a bridge and analyze noise information to determine whether there is damage to the bridge, then the specific position and degree of bridge damage and other information are obtained. 
The method of manual periodic inspection and measurement to obtain monitoring data is time-consuming and laborious, and it cannot meet the needs of real-time monitoring. In addition, contact sensors ${ }^{(3)}$ such as displacement meters, accelerometers, and other equipment are used to obtain signals for monitoring bridges, but the measuring range is small, and the equipment can easily be damaged and can cause damage to the bridges themselves. However, ground-based synthetic aperture radar (GBSAR) ${ }^{(4)}$ has the advantages of being noncontact, all-weather, and easy to operate, with high precision, high frame frequency, and so forth. As a high-precision deformation measurement instrument, ${ }^{(5)}$ GBSAR can achieve the real-time monitoring of small deformations of bridges. ${ }^{(6)}$

It is difficult to avoid an effect of the external environment when collecting bridge signals, resulting in noise in monitoring signals, ${ }^{(7)}$ which are not conducive to the identification of non-damage source signals. Therefore, before the identification of damage from the original monitoring data, blind source separation (BSS) technology can be used to separate the original mixed signals ${ }^{(8)}$ and suppress the noise signals unrelated to the damage. BSS refers to the process of separating all the original source signals that cannot be obtained directly by measurement from the mixture of an unknown number of observational signals. ${ }^{(9)}$ The blind source term here has two meanings: the number of source signals is unknown and the mixing mode of the source signals is unknown. Most monitoring signals have the above characteristics, so the BSS method has been widely applied and has attracted the attention of many scholars. ${ }^{(10)}$ Zhu et al. used the BSS method to identify sensor outliers as a bridge damage identification index. The results of a simulation experiment on the design of an aluminum bridge showed that this method could identify the abnormal changes in the bridge. ${ }^{(11)}$

The natural frequency of a bridge can be selected as an indicator for bridge damage identification, ${ }^{(12)}$ which has the advantage of easy measurement. Mode recognition can be used for this method. The test accuracy decreases when using the natural frequency, but the amount of information contained increases. ${ }^{(13)}$ In addition, the change in the flexibility matrix can also be used; ${ }^{(14)}$ this change is more sensitive to damage than the natural frequency and mode shape, but it has a flexibility matrix only in the lower modes. ${ }^{(15)}$

In view of the above situation, in this study, a BSS algorithm was combined with the change in bridge frequency to study bridge damage identification. The proposed method consisted of two main steps: (1) The second-order blind identification (SOBI) algorithm was used to separate the original monitoring signals of the bridge; (2) the fast Fourier transform (FFT) was used to calculate the separated and source signals to obtain the corresponding frequency and then compare the similarities and differences of the two signals. This method was expected to reduce the interference of other signal sources and improve the effectiveness of bridge damage identification. The study area, experimental data, and study methods are described in detail in Sect. 2. Then, the results and discussion of bridge damage identification are presented in Sect. 3. The conclusions of this study are given in Sect. 4 .

\section{Materials and Methods}

The bridge damage identification method adopted in this study mainly included two processing steps. In the first step, SOBI calculation was conducted on the bridge monitoring 
data. The second step was to take the FFT of the original data. Then, the frequencies of healthy and damaged bridges were obtained and compared. FFT was applied to the separate damaged and healthy bridge signals obtained from step one, and then the frequency was analyzed from the spectrum chart. Finally, the frequency of the separated signal calculated by SOBI was compared with that of the source signal to obtain the frequency change.

\subsection{Study area and data}

The raw measurement data used in this experiment were the monitoring signals of the Beishatan Bridge, Beijing, obtained with GBSAR. This bridge connects the G6 expressway between Beijing's north fourth ring and north fifth ring roads, as shown in Figs. 1(a)-1(d). The G6 highway, built in 1996, is one of the most important modes of land transportation in northwest China. The Beishatan Bridge consists of two sub-bridges, one on the right and the other on the left. The lengths and widths of the two sub-bridges are the same. To ensure the safe operation of the bridge, total-station instruments are used for regular monitoring, with three monitoring points selected along the cross section on the bridge surface. However, in a monitoring result, a maximum deviation of about $8 \mathrm{~cm}$ occurred at one point on the right side of the bridge. In order to avoid further deterioration of the right side of the Beishatan Bridge,

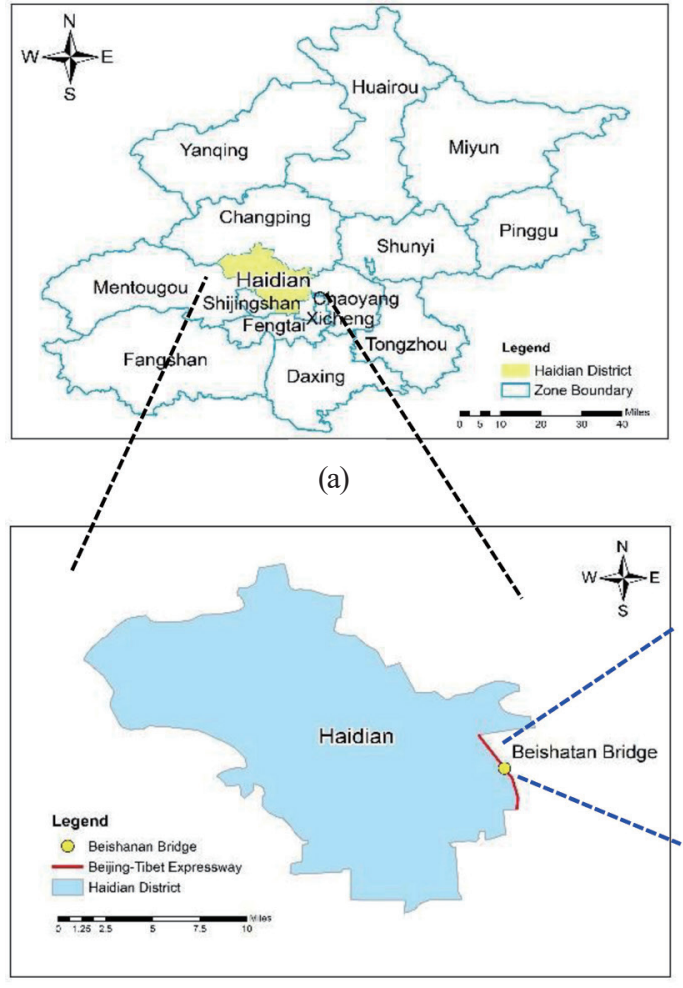

(b)

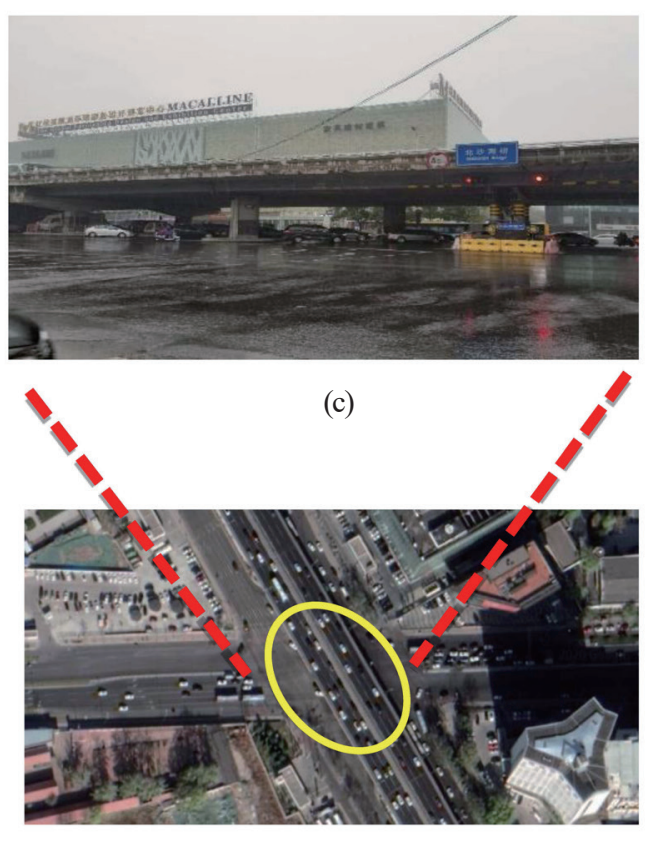

(d)

Fig. 1. (Color online) Location of study area. (a) Map of Beijing's region. (b) Specific location of study area. (c) Photograph of study area from Google Earth. (d) Field of photography of study area. 
its right side was supported by a jack during the study. Therefore, it was very easy to obtain images of the damage, to determine the reasons for the damage of the bridge, and to facilitate the reinforcement and maintenance of the bridge.

The original measurement data of the bridge in this experiment were obtained by simultaneously measuring the signals of the three adjacent points under the damaged bridge with a GBSAR instrument. The situation of signal acquisition is shown in Fig. 2. Details of the measured data are shown in Table 1. The total length of these data was $80 \mathrm{~s}$. The sampling frequency was $200 \mathrm{~Hz}$. The group of the healthy and damaged bridges measured 9048 data points. These measured data for the healthy bridge were also the signal of the three adjacent points under the bridge obtained simultaneously through GBSAR. The original measured data for the damaged and healthy bridges were processed in MATLAB after being processed through an xlsx file.

\subsection{Principle and algorithms of SOBI}

SOBI is a typical calculation method in a BSS algorithm that is calculated with secondorder statistics of samples. ${ }^{(16)}$ Its main feature is that the different delay information of signals is used to calculate the separation matrix of the best estimation according to the multiple timedelay covariance matrices of albino data. ${ }^{(17)}$ The second-order blind recognition algorithm was developed on the basis of the algorithm for multiple unknown signal extraction (AMUSE). AMUSE is calculated with the covariance matrix of two measurement vectors with different time delays. Additionally, SOBI calculates many covariance matrices without a delay. This solves the problems of difficulty in selecting the delay, robustness against noise, and poor equal performance, and AMUSE has universality. ${ }^{(18)}$

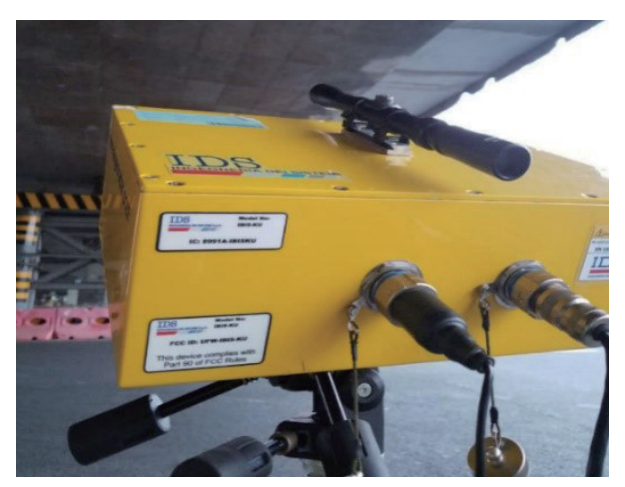

Fig. 2. (Color online) GB-SAR monitoring site.

Table 1

Information of measured signals.

\begin{tabular}{lccc}
\hline Sensor (Type) & Data & Time (CST) & Temporal resolution (s) \\
\hline \multirow{2}{*}{ GB-SAR (Ibis-s) } & Signal of healthy bridge & June 2017 & 0.008 \\
\cline { 2 - 4 } & Signal of damaged bridge & July 2017 & 0.008 \\
\hline
\end{tabular}


The main idea of the SOBI algorithm is to use matrix diagonalization to solve the separation matrix and then obtain the separation source signal. ${ }^{(19)}$ Therefore, the key step of this algorithm is to find the orthogonal matrix $V$ that can diagonalize the covariance matrix of different delays. A schematic diagram of SOBI is shown in Fig. 3.

The main steps of the SOBI algorithm are as follows. First, the acquired raw measured signal is preprocessed. This step involves de-averaging the raw observed signal. This calculation is performed to simplify the raw numbers. Then, the signal is whitened by a singular matrix to remove the correlation between signals. Next, the covariance matrix of the albino signal with delay $p$ is calculated, and the joint approximate diagonalization algorithm is used to diagonalize the covariance matrix of different time delays. ${ }^{(20)}$ Then, an orthogonal matrix is obtained. Finally, the best estimations of the mixed matrix and source signal are calculated accordingly from the orthogonal and albino matrices.

The algorithm flow of SOBI is as follows. It is assumed that the number of known observed signals is $m: X(t)=\left[x_{1}(t), x_{2}(t), \ldots, x_{n}(t)\right]^{T}$, the number of unknown source signals is $n: S(t)=\left[s_{1}(t), s_{2}(t), \ldots, s_{n}(t)\right]^{T}$, and the number of albino signals is $n: Z(t)=\left[z_{1}(t), z_{2}(t), \ldots, z_{n}(t)\right]^{T}$. In the first step, the observed signals are de-averaged, and then the covariance is calculated using the de-averaged data, where $n$ is the number of maximum eigenvalues represented by $\lambda_{1}, \lambda_{2}, \ldots, \lambda_{n}$, and their corresponding eigenvectors are $h_{1}, h_{2}, \ldots, h_{n}$. Second, the maximum eigenvalue, the corresponding eigenvector, and the average of the $m-n$ minimum eigenvalues of the covariance of the measured data are used to calculate an $n \times m$-dimensional albino matrix $W$ :

$$
W=\left[\left(\lambda_{1}-\sigma^{2}\right)^{-1 / 2} h_{1}, \ldots,\left(\lambda_{n}-\sigma^{2}\right)^{-1 / 2} h_{n}\right]^{T} .
$$

Then, albino processing is carried out on the albino matrix and observed signals to obtain the albino data $Z(t)$ :

$$
Z(t)=W \times X(t) .
$$

The mathematical model of the linear instantaneous mixing of signals can be expressed as $x(t)=A \times s(t)$, where $x(t)$ is the matrix of the observed signals, $A$ is an $m \times n$ mixing matrix, and $s(t)$ is the matrix of the source signal. Therefore, $Z(t)$ can be expressed as

$$
Z(t)=W \times A \times S(t)=V \times S(t),
$$

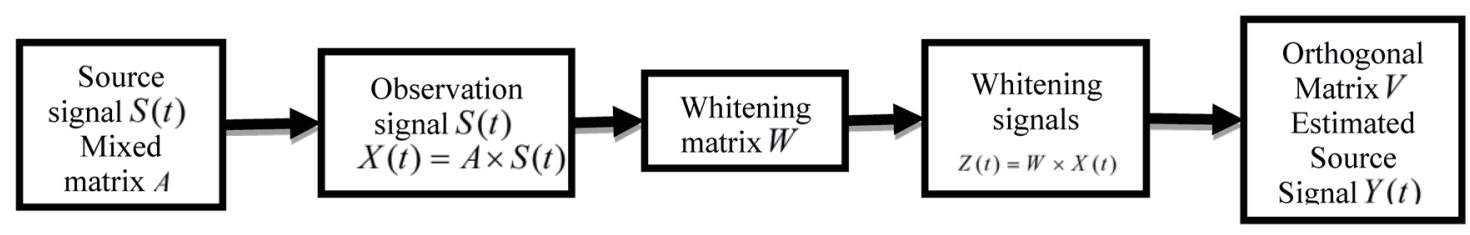

Fig. 3. Flow of SOBI signal processing. 
where $V$ is an orthogonal matrix. Then, at a fixed delay time $\tau \in\left\{\tau_{i} \mid i=1,2, \ldots, k\right\}$, the albino covariance matrix $R(\tau)$ is calculated as

$$
R(\tau)=E\left[Z(t) Z^{T}(t+\tau)\right]=A R_{Z}(\tau) A^{T}
$$

where $R(\tau)$ are computed with the joint approximate diagonalization algorithm, and then the orthogonal matrix $V$ is obtained. Finally, the mixing matrix $A$ is estimated as $A=W^{-1} V$. The best estimate of the source signal is $S(t)=V^{T} W X(t)$.

\subsection{FFT}

FFT is an algorithm that transforms a signal from the time domain to the frequency domain. It is a polynomial product and a fast algorithm for a discrete Fourier transform (DFT). The advantage of this method is that its time complexity is lower than that of DFT, thus reducing the computation time, ${ }^{(21)}$ and the method is convenient and easy to operate when processing signals.

FFT decomposes a Fourier transform into the product of several simple matrices and uses the idea of a bisector to transform the time and frequency domains. FFT originates from DFT; DFT is defined as

$$
X(\kappa)=\sum_{n=0}^{N-1} x(n) e^{-i \frac{2 \pi}{N} n \kappa}, k=0,1, \ldots, N-1
$$

where $X(\kappa)$ is the frequency-domain signal and $x(n)$ is the time-domain signal. ${ }^{(22)}$

The main characteristic of FFT is that its computation speed is higher than that of DFT. The most important point is that the time-domain signal can be converted to the frequency-domain signal, and the frequency recognition has good accuracy. Therefore, in this study, the frequency recognition of each separated signal was accomplished by SOBI and FFT.

The flow chart of this study is shown in Fig. 4. There were four main steps in this study: the preprocessing of the measured signal, the calculation of separated signal, the identification of the frequency, and the comparison of the frequencies of the results.

\section{Results and Discussion}

\subsection{Simulation experiment of analog signal frequency recognition}

To verify the effect of the SOBI method in separating signals to reduce noise interference and to apply the method to bridge damage identification, in this study, a simulation experiment of SOBI and separation was conducted using a group of single-frequency analog signals and a group of single-double-frequency mixed analog signals, as well as an experiment based on the real monitoring data of the Beishatan Bridge in Beijing.

The damage identification method based on BSS was simulated by a group of analog signals with a single frequency and another group of mixed analog signals with single 


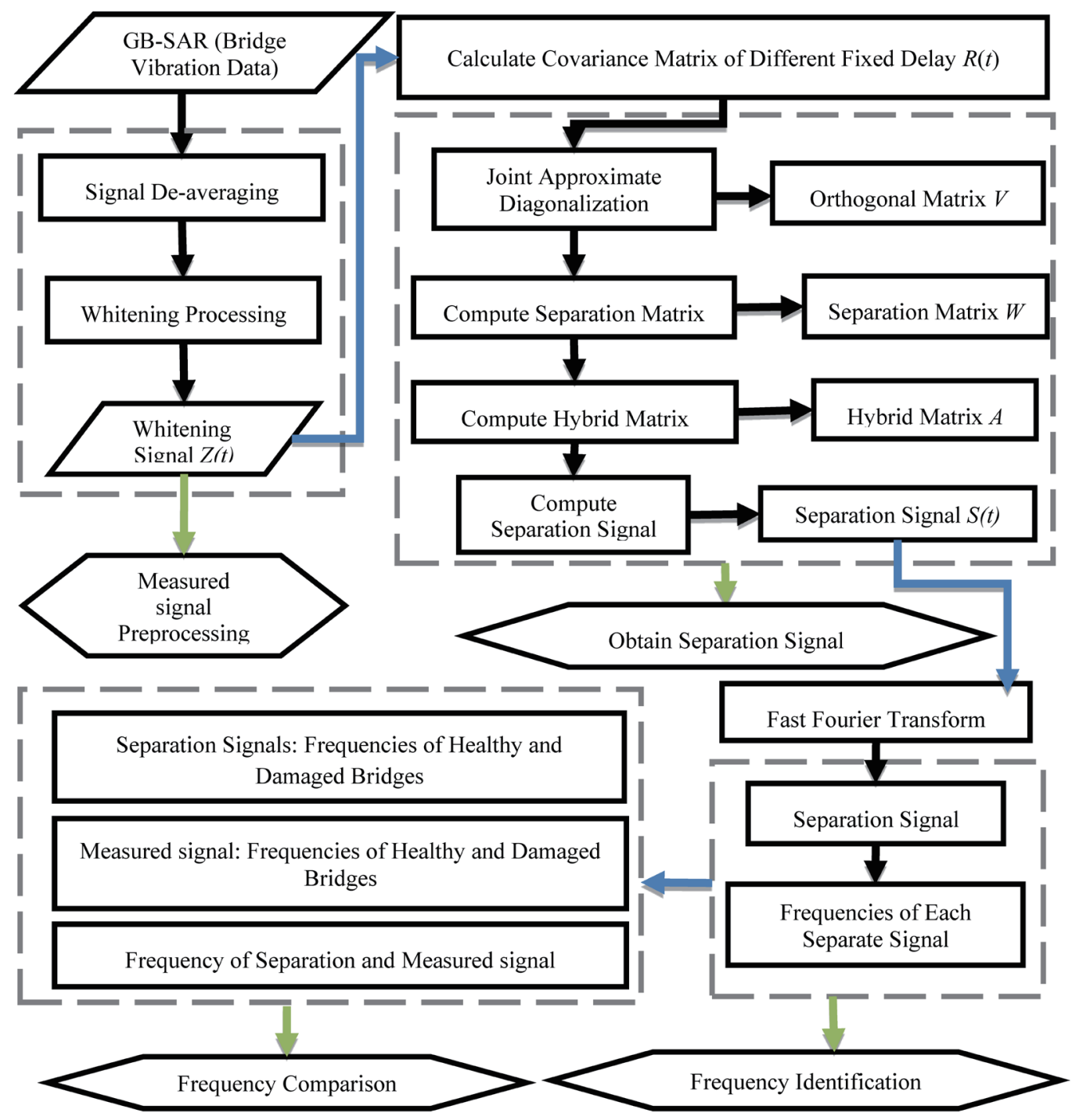

Fig. 4. (Color online) Flow of SOBI signal processing.

and double frequencies. The first group of analog signals was as follows: source signal 1 , $s_{1}=7 \times \sin \left(2 \times \pi \times f_{1} \times t\right)$, source signal $2, s_{2}=2+3 \times \sin \left(2 \times \pi \times f_{2} \times t\right)$, and source signal 3, $s_{3}=2 \times \sin \left(2 \times \pi \times f_{3} \times t\right)$. The theoretical frequencies were $f_{1}=0.05, f_{2}=3$, and $f_{3}=0.5 \mathrm{~Hz}$. The preset sampling frequency $F_{S}$ was $200 \mathrm{~Hz}$, which was the same as that of GBSAR when the bridge data were acquired. In addition, the signal length $N$ was set to 5000. According to the sampling frequency and signal length, the time was $t=0: 1 / F_{S}:(n-1) / F_{S}$. The following mixing matrix, randomly generated using MATLAB, was used to mix the three simulated source signals:

$$
H=\left(\begin{array}{lll}
0.8147 & 0.9134 & 0.2785 \\
0.9058 & 0.6324 & 0.5469 \\
0.1270 & 0.0975 & 0.9575
\end{array}\right)
$$


MATLAB was used to draw the source signal of the analog signal, as shown as Fig. 5(a). The mixed signals are shown in Fig. 5(b). The signals separated by the SOBI algorithm are shown in Fig. 5(c). The frequency domain signals obtained by FFT are shown in Fig. 5(d).

It can be seen from the simulated source and separated signals that the amplitude of the separated signals differed from that of the source signals owing to the insufficient data. However, the waveform of the signals, as well as their frequency, remained constant. In practice, the mixed signals were more complex, and it was difficult to directly identify the parameters and characteristics.

We performed error analysis by comparing the theoretical and detected frequencies, as shown in Table 2.
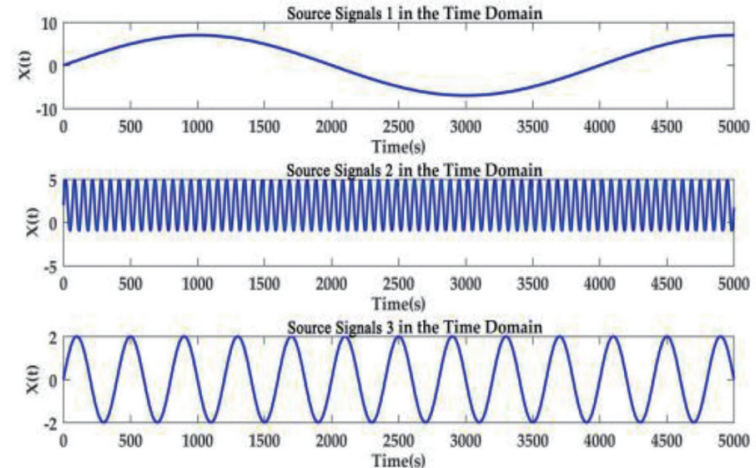

(a)
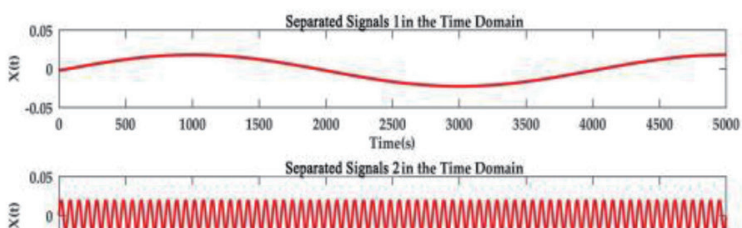

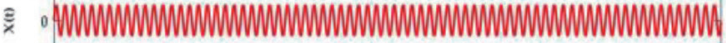
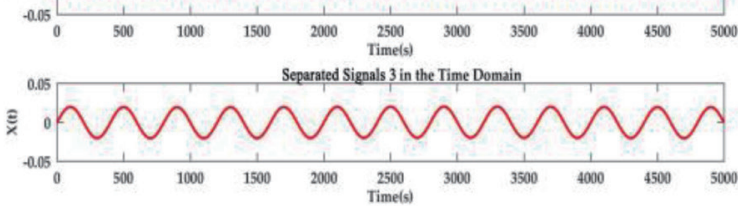

(c)
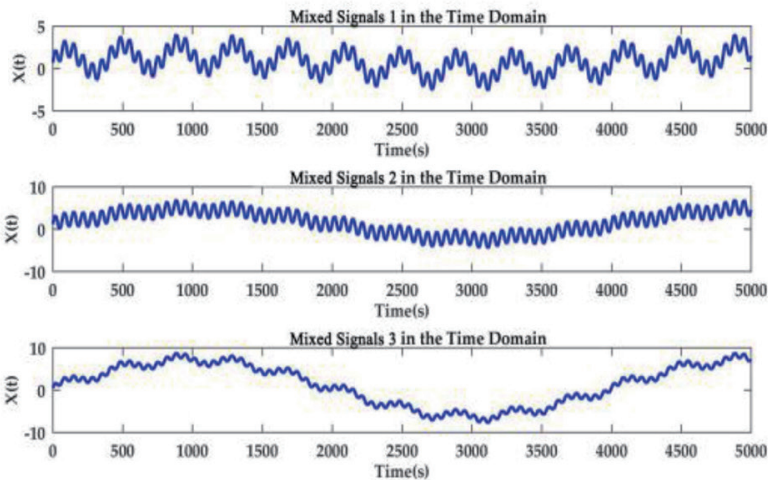

(b)
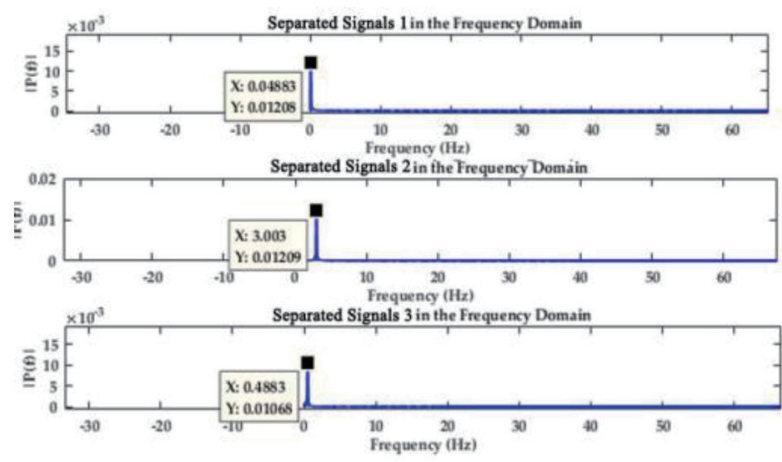

(d)

Fig. 5. (Color online) Simulation experiment 1. (a) Source signals in time domain. (b) Mixed signals in time domain. (c) Separated signal in time domain. (d) Frequency domain signals in time domain.

Table 2

Frequency identification results of single-frequency signal.

\begin{tabular}{lcccc}
\hline $\begin{array}{l}\text { Number of } \\
\text { separated signal }\end{array}$ & $\begin{array}{c}\text { Theoretical } \\
\text { frequency }(\mathrm{Hz})\end{array}$ & $\begin{array}{c}\text { Identification } \\
\text { frequency }(\mathrm{Hz})\end{array}$ & Error $(\mathrm{Hz})$ & $\begin{array}{c}\text { Relative error } \\
(\%)\end{array}$ \\
\hline 1 & 0.05 & 0.04883 & 0.00117 & 2.34 \\
2 & 3 & 3.003 & 0.003 & 0.1 \\
3 & 0.5 & 0.4883 & 0.0117 & 2.34 \\
\hline
\end{tabular}


It was found from the recognition results of the single-frequency signal in Table 1 that the frequency obtained by FFT was accurate to one or two decimal places. The relative error of the recognition frequency of separated signal 2 was small, so FFT accurately detected the frequency of the separated signal.

Because the frequency of the monitored bridge signal was low and that of the separated signal was not a single value, a simulation experiment 2 was designed to verify the effect of the combined methods of BSS and FFT on the accuracy of detecting the frequency. In this experiment, two dual-frequency source signals and one single-frequency source signal formed by the combination of sine and cosine waves were selected as follows: source signal 1 was $s_{1}=7 \times \sin \left(2 \times \pi \times f_{1} \times t\right)+3 \times \cos \left(2 \times \pi \times f_{2} \times t\right)$, source signal 2 was $s_{2}=2+3 \times \sin \left(2 \times \pi \times f_{3} \times t\right)+2 \times \sin \left(2 \times \pi \times f_{4} \times t\right)$, and source signal 3 was $s_{3}=7 \times \sin \left(2 \times \pi \times f_{5} \times t\right)$. The theoretical frequencies of the three signals used in the simulation experiment 2 were $f_{1}=0.05$, $f_{2}=0.1, f_{3}=0.3, f_{4}=0.4$, and $f_{5}=0.5 \mathrm{~Hz}$. Corresponding to the sampling frequency of the real data experiment, the sampling frequency $F_{S}$ remained unchanged at $200 \mathrm{~Hz}$. The variables of simulation experiment 2 were the number of separated signal and the frequencies. Therefore, to enable a comparison with simulation experiment 1 , the signal length $\mathrm{N}$ was maintained constant at 5000 and the time was set to $t=0: 1 / F_{S}:(n-1) / F_{S}$. The following randomly generated mixing matrix was used to simulate the real mixed signals:

$$
H=\left(\begin{array}{lll}
0.8308 & 0.9172 & 0.7537 \\
0.5853 & 0.2858 & 0.3804 \\
0.5497 & 0.7572 & 0.5678
\end{array}\right)
$$

This matrix was similar to that generated in simulation experiment 1 . In simulation experiment 2, the simulation signal was input to MATLAB, and the source signal in the time domain [Fig. 6(a)] was drawn with the BSS and FFT algorithms. The random mixed signal is shown in Fig. 6(b), the separated signal in the time domain is shown in Fig. 6(c), and the separated signal in the frequency domain is shown in Fig. 6(d).

By comparing the waveform and numerical analysis results of the separation and source signal graphs in simulated experiment 2, SOBI was found to be less effective at recognizing multifrequency signals than at recognizing single-frequency signals. As can be seen from the intercept of the centralized frequency information, as shown in Fig. 6(d), the recognition frequencies of separation signal 1 were 0.04883 and $0.09766 \mathrm{~Hz}$, and the theoretical frequencies were 0.05 and $0.1 \mathrm{~Hz}$.

The first frequency of separation signal 1 was consistent with the recognition result of the theoretical frequency of $0.05 \mathrm{~Hz}$ in simulation experiment 1 . The recognition frequencies of separated signal 2 were 0.293 and $0.3906 \mathrm{~Hz}$, and the corresponding source signal 2 theoretical frequencies were 0.3 and $0.4 \mathrm{~Hz}$; the recognition frequencies were slightly lower than the theoretical frequencies. Isolated signal 3 was a single-frequency signal and its recognition frequency was $0.4883 \mathrm{~Hz}$. The theoretical frequency of source signal 3 was $0.5 \mathrm{~Hz}$, which was consistent with the recognition frequency corresponding to the same theoretical frequency in the first group of experiments. 

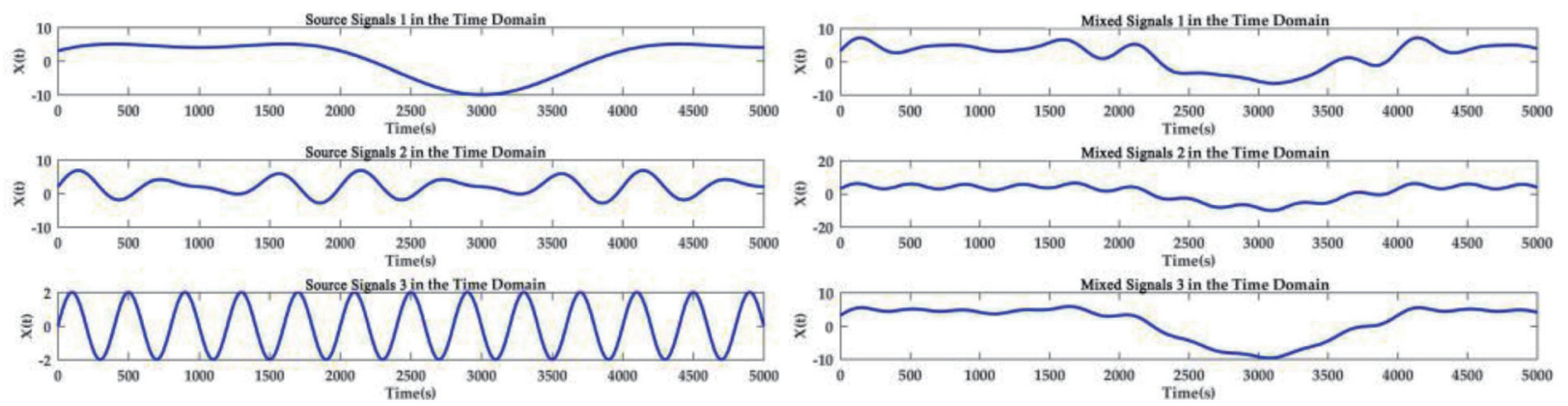

(a)

(b)
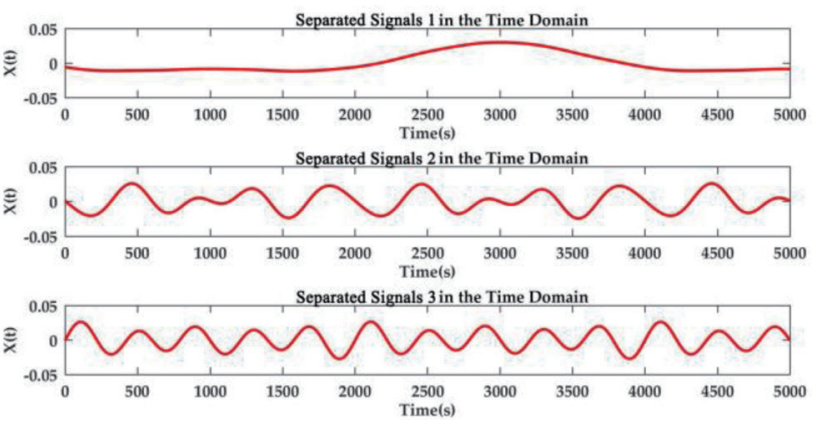

(c)
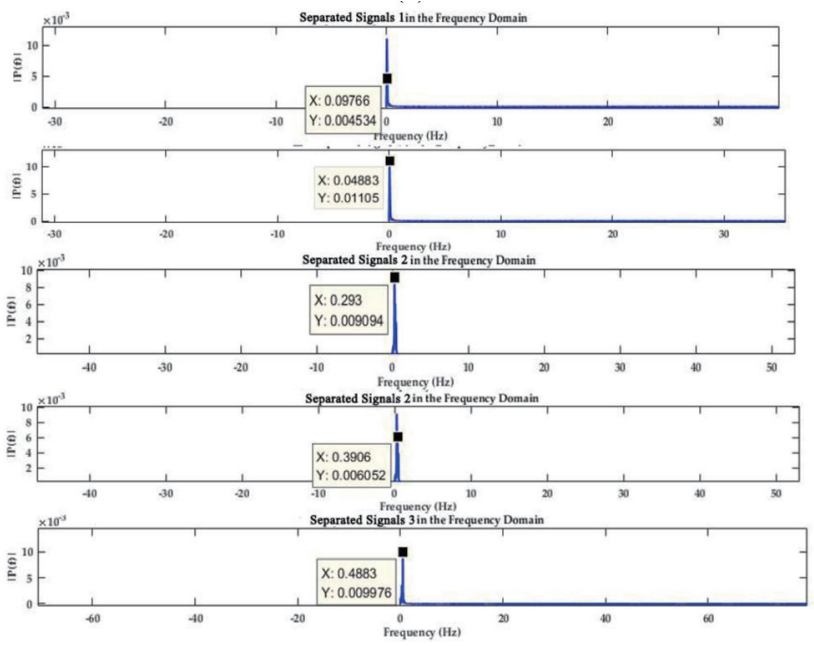

(d)

Fig. 6. (Color online) Simulation experiment 2. (a) Source signals. (b) Mixed signals. (c) Separated signal. (d) Frequency domain signals.

By comparing the theoretical and recognition frequencies of the signals in the second group of simulation experiments, the error analysis results were obtained and are shown in Table 3. It can be seen from Table 3 that the relative errors of the frequency recognition of the three analog signals in the second group of simulation experiments ranged between 2.3 and $2.4 \%$. Therefore, it can be seen that the recognized frequencies of the single- and multifrequency mixed signals were accurate to one or two decimal places. Therefore, the SOBI and FFT algorithms can be used to identify the frequency of low-frequency multifrequency signals.

\subsection{Field verification experiment for Beishatan Bridge}

Through the above two sets of simulation experiments for the single-frequency signal and single- and double-frequency mixed signal, it was concluded that the separated signal can be obtained with the SOBI algorithm, and then the frequency of a signal could be obtained with relatively high accuracy by the frequency domain analysis of the FFT algorithm. Next, the GBSAR equipment was used to conduct field measurements and obtain the bridge data for the two states of health and damage. 
Table 3

Frequency identification results of multifrequency signals.

\begin{tabular}{lcccc}
\hline $\begin{array}{l}\text { Separated } \\
\text { signal }\end{array}$ & $\begin{array}{c}\text { Theoretical } \\
\text { frequency }(\mathrm{Hz})\end{array}$ & $\begin{array}{c}\text { Identification } \\
\text { frequency }(\mathrm{Hz})\end{array}$ & $\begin{array}{c}\text { Error } \\
(\mathrm{Hz})\end{array}$ & $\begin{array}{c}\text { Relative error } \\
(\%)\end{array}$ \\
\hline 1 & 0.05 & 0.04883 & 0.00117 & 2.34 \\
\hline 2 & 0.1 & 0.09766 & 0.00234 & 3.34 \\
\hline 3 & 0.3 & 0.293 & 0.007 & 2.33 \\
\hline
\end{tabular}
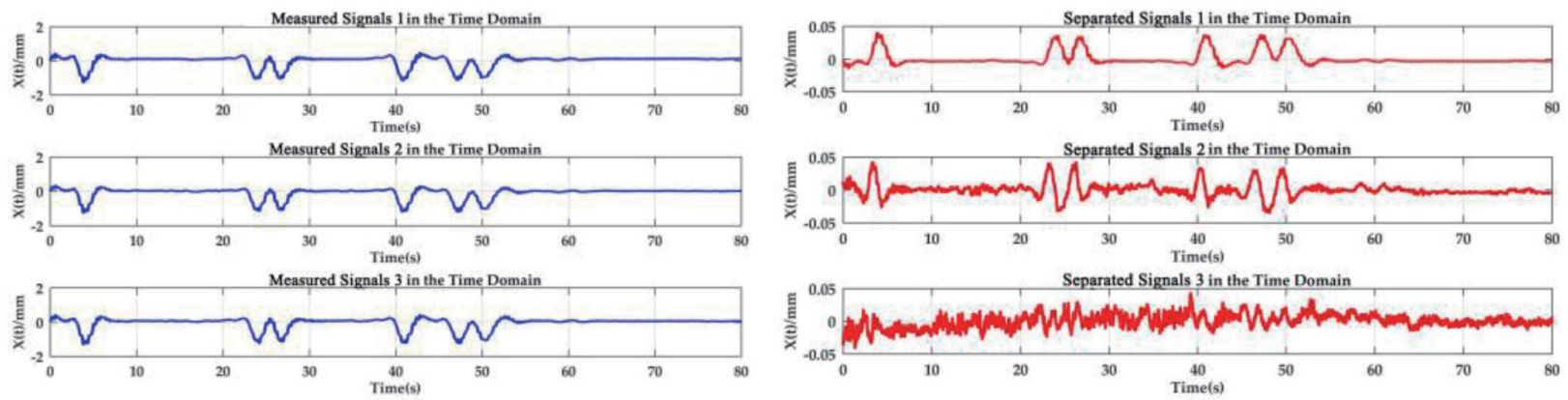

(a)

(b)
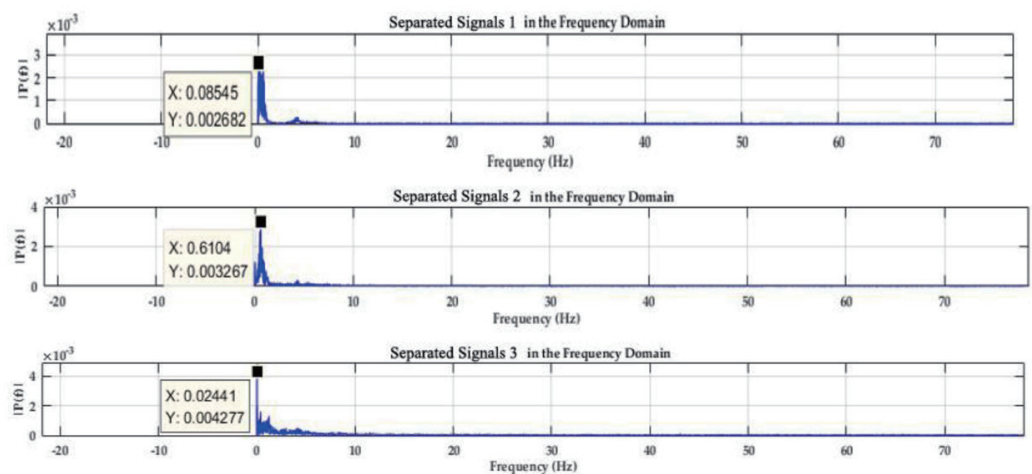

(c)

Fig. 7. (Color online) Signals of damaged bridge. (a) Measured signals. (b) Separated signals in time domain. (c) Separated signals in frequency domain.

First, the bridge damage was located when the data were read in MATLAB and the time displacement was mapped out. The measured signals from the damaged bridge were obtained, as shown in Fig. 7(a), with three adjacent points for vibration signals.

After calculation using the SOBI algorithm, the separation signal graph was obtained and is shown in Fig. 7(b). It can be seen from the graph that the first signal was more stable than the second and third separated signals. The displacement in the measured signal showed a negative increase, while that in separated signal 1 showed a positive increase; the displacement change of separated signal 1 occurred at the same time as that of the source signal. Separated signal 2 of the damaged bridge also showed a positive increase in displacement, which occurred about $1 \mathrm{~s}$ earlier than that of separated signal 1. In addition, the waveform of separated signal 2 was less 
stable than that of separated signal 1 at other times. At the time of damage, the waveform of separated signal 3 exhibited a large vibration because there was a small change in displacement at $80 \mathrm{~s}$, and it was impossible to distinguish when the displacement changed significantly. Therefore, signal 3 is considered to represent other signals unrelated to the source of damage.

Through the waveform and numerical analyses of the three separated signals, it was concluded that the BSS algorithm can separate other signals that interfered with the signal of the bridge itself. Then, the frequency of each separated signal was obtained by the FFT of the separated signals in Fig. 7(c), as shown in the frequency domain diagram of the separated signals in the case of damage. It could be seen that the frequencies of the bridge were relatively low, in agreement with the theoretical frequencies obtained in the above simulation experiment.

For the healthy bridge measurement data input, MATLAB was used simultaneously with GBSAR to obtain the time displacement data of three monitoring sites in order to map out the measured signals from the healthy bridge, as shown in Fig. 8(a). There was an obvious increase in the displacement of the negative change of the three monitoring signals at 26.9 s. However, the measured signal of the healthy bridge at $40-43 \mathrm{~s}$ for time 2 appeared to have irregular vibration, and measured signal 3 at $40-46 \mathrm{~s}$ appeared to also have irregular vibration but lasted longer than measured signals 2 and 1. All the three measured signals showed a negative increase in displacement at $67.3 \mathrm{~s}$, which was smaller than that at $26.9 \mathrm{~s}$. The change in displacement at that time was about half of that at $26.9 \mathrm{~s}$. The waveforms of the other three
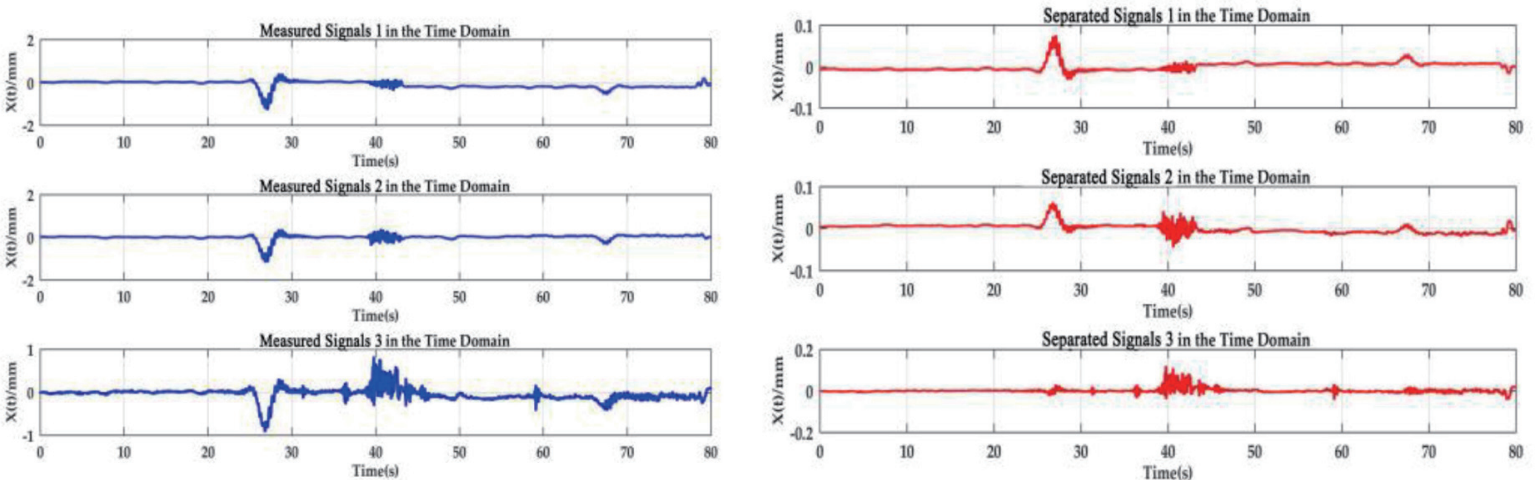

(a)

(b)
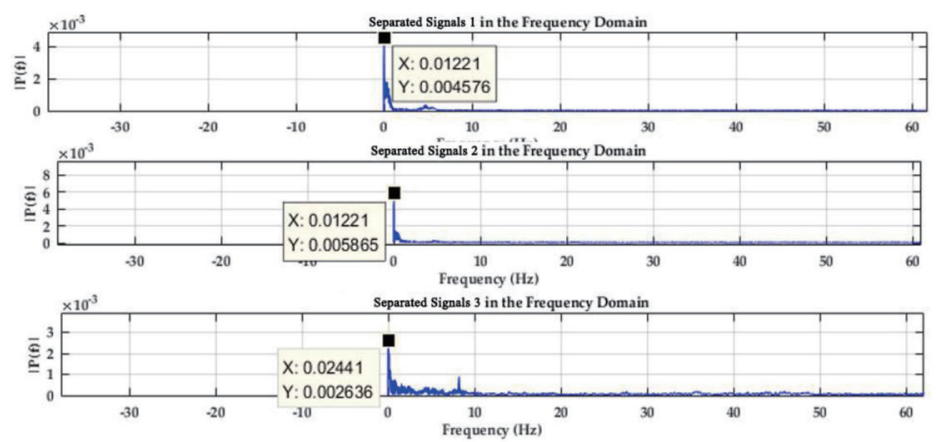

(c)

Fig. 8. (Color online) Signals of healthy bridge. (a) Measured signals. (b) Separated signals in time domain. (c) Separated signals in frequency domain. 
signals were relatively stable, but the waveform of measured signal 3 had a larger vibration amplitude than those of measured signals 1 and 2. Then, the measured signals of the healthy bridge were separated with the SOBI algorithm, and the separated signals shown in Fig. 8(b) were obtained. It can be seen that separation signal 1 of the healthy bridge had positive displacement changes of $0.07 \mathrm{~mm}$ at $27 \mathrm{~s}$ and $0.025 \mathrm{~mm}$ at $68 \mathrm{~s}$. There was a small signal vibration in the period of 39-43 s. The difference between separation signal 1 and measured signal 1 was that the displacement became positive, and the signal waveform of the rest of separation signal 1 was more stable than that of measured signal 1. Separation signal 2 showed a positive displacement change of $0.06 \mathrm{~mm}$ at $26.87 \mathrm{~s}$, which was smaller than that of separation signal 1, while the displacement of separation signal 2 at $68 \mathrm{~s}$ was $0.03 \mathrm{~mm}$, roughly the same as that of separation signal 1. The time of signal vibration also occurred in the period of 39-43 s, and the vibration amplitude was greater than that of separation signal 1. Separation signal 3 had no obvious change in displacement, but in the period of 40-47 s, it showed a larger waveform vibration than signals 1 and 2 . Thus, we consider that separation signal 3 of the healthy bridge was the separated noise signal.

The three separated time-domain signals of the healthy bridge were transferred to the frequency domain by FFT calculation. The spectrum diagram shown in Fig. 8(c) was drawn in MATLAB. The frequency maps obtained from separated signals 1 and 2 were used to determine that the frequency of both signals was $0.01221 \mathrm{~Hz}$ and that the frequency of separation signal 3 was $0.02441 \mathrm{~Hz}$.

By recording the frequencies obtained under the damage and healthy conditions, we compared the frequency increments of the three separated initial signals of the healthy and damaged bridges, as shown in Table 4. It can be seen that the frequencies of separated signals 1 and 2 for the healthy bridge were consistent, both of which were $0.1221 \mathrm{~Hz}$. The frequencies of separated signals 1 and 2 for the damaged bridge were greater than those for the healthy bridge, and the frequency of separation signal 2 for the damaged bridge was higher than that of separation signal 1 . This shows that the frequency of separation signal 2 was most sensitive to damage, indicating that this signal is the most suitable for identifying damage. The frequency of separation signal 3 for the healthy and damaged bridges did not change, so the frequency of separation signal 3 was concluded to be independent of the damage of the bridge, verifying that separation signal 3 was unrelated to the damage.

From the relationship between the measured and separated signals, the waveform of separated signal 1 was found to be more stable than that of separated signal 2. However, from the frequency increment, the increment of separation signal 2 was determined to be greater, so the signal most suitable for damage recognition cannot be determined by the waveform alone.

Table 4

Frequencies of separated signal for healthy and damaged bridges.

\begin{tabular}{lccc}
\hline $\begin{array}{l}\text { Separated } \\
\text { signals }\end{array}$ & $\begin{array}{c}\text { Frequency of } \\
\text { healthy bridge }(\mathrm{Hz})\end{array}$ & $\begin{array}{c}\text { Frequency of } \\
\text { damaged bridge }(\mathrm{Hz})\end{array}$ & $\begin{array}{c}\text { Increase } \\
(\%)\end{array}$ \\
\hline 1 & 0.01221 & 0.08545 & 7.324 \\
2 & 0.01221 & 0.6104 & 59.819 \\
3 & 0.02441 & 0.02441 & 0 \\
\hline
\end{tabular}




\subsection{Effect of SOBI on damage identification of the bridge}

To verify the effectiveness of the SOBI algorithm for bridge damage identification, a group of comparative experiments was designed in this study. The measured signals used to evaluate the healthy and damage conditions of the bridge were directly identified with FFT, and the frequency results of the two methods were compared. The frequency domain diagram obtained from the monitoring signal of the original damaged bridge is shown in Fig. 9(a). In the same manner, the frequency graphs of three healthy bridge measured signal were obtained and are shown in Fig. 9(b), and the frequencies of these two groups were recorded.

The bridge frequencies obtained under damage and healthy conditions were recorded. The measured signal frequencies are listed in Table 5. Among the measured points from the healthy bridge, the recognition frequencies of the measured signals at two points were both $0.01221 \mathrm{~Hz}$, and the frequency of the measured signals at one point was $0.2563 \mathrm{~Hz}$, which was significantly different from the other two values. In the three-measurement-point set from the damaged part of the bridge, the frequencies of the measured signal obtained from the two points were both $0.08545 \mathrm{~Hz}$. The recognition frequency of the measured signal at the other point was $0.01221 \mathrm{~Hz}$, which was the same as that obtained under the healthy condition. Moreover, the recognition frequencies of measured signals 1 and 3 were the same under the two conditions, so they can be regarded as the frequencies of the bridge when it was healthy and damaged. However, the frequency of measured signal 2 was considerably different from the other two values for the healthy condition. In addition, both measured signals 1 and 3 had higher frequencies during injury, while measured signal 2 showed a significant decrease in frequency.
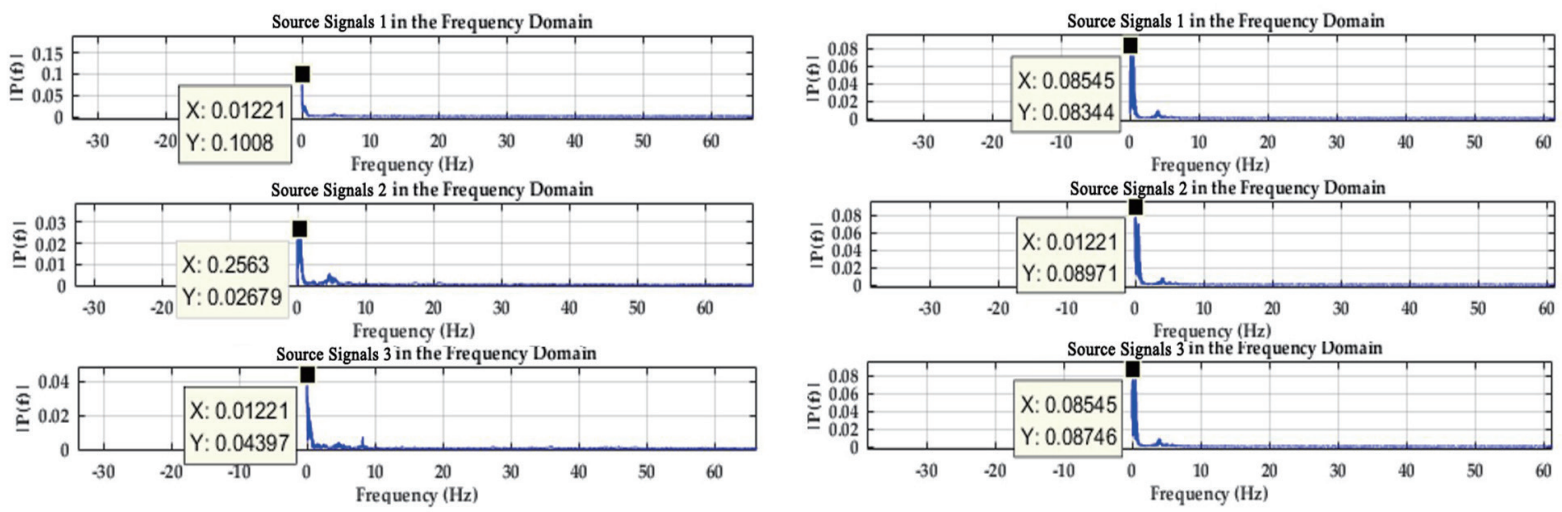

(a)

(b)

Fig. 9. (Color online) Results in frequency domain. (a) Damaged bridge measured signal in frequency domain. (b) Healthy bridge measured signal in frequency domain.

Table 5

Frequencies of separated signal for healthy and damaged bridges.

\begin{tabular}{lccc}
\hline Measured signal & Health frequency $(\mathrm{Hz})$ & Damaged frequency $(\mathrm{Hz})$ & Increase $(\%)$ \\
\hline 1 & 0.01221 & 0.08545 & 7.324 \\
2 & 0.2563 & 0.01221 & - \\
3 & 0.01221 & 0.08545 & 7.324 \\
\hline
\end{tabular}


From the identified frequencies shown in Tables 4 and 5, two conclusions can be drawn. One conclusion was that measured signal 2 in the healthy and damaged parts of the bridge had a frequency that was different from those of the other two signals, and separated signal 2 calculated with the SOBI algorithm was the signal whose frequency increased the most. The other conclusion was that the frequency of separation signal 3 was the same for the healthy and damage conditions, indicating that it was unrelated to the damaged bridge. The anomalies in measured signal 2 may have been caused by some interference signals, rather than the frequency attribute of the bridge itself. In this manner, the advantages of the BSS algorithm can be seen, that is, the accuracy of the data increased, the recognition result was reliable, and the damage condition of the bridge was clearly determined. Furthermore, by combining the SOBI algorithm with the FFT calculation, a more accurate determination of the damage condition of the bridge can be obtained.

\section{Conclusions}

In this study, the SOBI algorithm in BSS technology was combined with the FFT method for bridge damage identification. This combination effectively reduced the interference of the noise signal, enhanced the signal associated with bridge damage, and improved the accuracy of bridge damage identification from the frequency. The BSS technology combined with the FFT method was suitable for identifying the signal collected from the bridge with noise present in the surrounding environment.

Through comparative experiments, the damaged part of the bridge was found to increase the frequency of $59.8 \%$ after separation using the SOBI algorithm, and without damage to the bridge, the frequency of the white noise signals of the healthier bridge increased by $7.3 \%$. Thus, for bridge damage identification, the impact of change in frequencies cannot be ignored. The SOBI of the signal separation technology was simple, feasible, and effective, and FFT can be used to rapidly and conveniently obtain the frequencies of the bridge to facilitate data analysis.

However, it was found from the measurement data experiment for the Beishatan Bridge that the bridge frequency was low and the frequency domain was not clearly recognized. Therefore, the short-time Fourier transform can be used for time-frequency analysis, and the effective frequency interval can be increased to obtain better results. In addition, the program should be improved to integrate the processes of signal separation, signal frequency extraction, and frequency contrast, as well as to enhance the automation of bridge damage identification and further improve the efficiency of bridge damage identification. Additionally, we conducted the experiment on only one bridge, so more tests should be carried out for other bridges.

We used the SOBI algorithm to separate the bridge signals. By separating the signals, we found that the change in significant frequency can be used to identify a damaged bridge. In future studies, the algorithm introduced in this study could be combined with sensors to obtain real-time bridge frequency data, effectively maintain a damaged bridge, and reduce financial losses and casualties. 


\section{Acknowledgments}

This study was sponsored by the National Natural Science Foundation of China (grant no. 41871367), the Ministry of Science and Technology of the People's Republic of China (grant no. 2018YFE0206100), General Scientific Research Project of Beijing Educational Committee (grant Nos. KM20190016006 and KM201910016007), Beijing Advanced Innovation Center for Future Urban Design, Beijing University of Civil Engineering and Architecture (grant no. UDC2019031321), and basic scientific research business expenses of municipal universities (grant no. X20150).

\section{References}

1 S . Hong: TranspoWorld 19 (2019) 49. https://doi.org/10.16248/j.cnki.11-3723/u.2019.19.049

2 D. L. Cheng, A. L. Yu, X. Y. Zhou, and G. J. Fan: Modern Electron. Tech. 7 (2019) 34. https://doi.org/10.16652/ j.issn.1004-373x.2019.07.032

3 Y. G. Chen and Z. Y. Zhong: J. Vibration Eng. 3 (2019) 12. https://doi.org/10.16385/j.cnki.issn.10044523.2019.03.012

4 Z. S. Huang, J. P. Sun, Q. Li, W. X. Tan, P. P. Huang, and Y. L. Qi: Sensors 18 (2018) 3883. https://doi. org $/ 10.3390 / \mathrm{s} 18113883$

5 Y. K. Deng, Q. Yuan, C. Hu, W. M. Tian, and Z. Zhao: J. Signal Process. 34 (2018) 1269. https://doi. org/10.16798/j.issn.1003-0530.2018.11.001

6 P. Guo, H. Y. Zhang, L. Chen, K. Q. Zhou, and C. L. Wang: Bulletin Surv. Mapp. 6 (2017) 94. https://doi. org/10.13474/j.cnki.11-2246.2017.0198

7 T. Yu, W.S. Hu, J. Wu, H.F. Li, and Y. Qiao: J. Vibration Shock 12 (2019) 38. https://doi.org/10.13465/j.cnki. jvs.2019.12.038

8 Y. Y. Tian, S. F. Ou, Y. Gao, and Q. Q. Jiang: Procedia Comput. Sci. 154 (2019) 524. https://doi.org/10.1016/ j.procs.2019.06.082

9 C. Y. Shen and Z. L. Liu: Commun. Technol. 12 (2018) 5. https://doi.org/10.3969/j.issn.1002-0802.2018.12.005

10 K.Wang, H.Cheng, and K.Zhang: Bulletin Sci. Technol. 2 (2019) 28. https://doi.org/10.13774/j.cnki. kjtb.2019.02.028

11 Y. J. Zhu, A. Jesus, I. Laory, and Y. Ren: Struct. Health Monit. (2017) 527. https://doi.org/10.12783/ shm2017/13907

12 Y. Q. Zhang, Y. Miyamori, T. Kadota, and T. Saito: Sens. Mater. 29 (2017) 165. https://doi.org/10.18494/ SAM.2017.1421

13 X. K. Shen: Technol. Highway Transport 32 (2016) 70. https://doi.org/10.13607/j.cnki.gljt.2016.05.017

14 S. H. Tang, N. Zhou, Z. Fang, and B. J. Su: Earthquake Eng. Eng. Dynam. 6 (2017) 11. https://doi.org/10.13197/ j.eeev.2017.06.98.tangsh.011

15 S. Lin, W. H. Li, and S. C. Zhao: J. Jilin Univ. 1 (2019) 9 (in Chinese). https://doi.org/10.13229/j.cnki. jdxbgxb20180389

16 Y. M. Bai, J. Z. Jiang, S. G. Liu, and Y. M. Sun: J. Data Acquisition Process. 5 (2016) 25. https://doi. org/10.16337/j.1004-9037.2016.05.025

17 B. H. Yang, Y. Y. Zhang, H. R. Li, T. Zhang, and K. W. Duan: J. Shanghai Jiao Tong Univ. 12 (2015) 24 (in Chinese). https://doi.org/10.16183/j.cnki.jsjtu.2015.12.024

18 Y. C. Kuo, C. T. Tsai, and C. H. Chang: Sens. Mater. 29 (2017) 713. https://doi.org/10.18494/SAM.2017.1487

19 Y. Y. Zhang, B. H. Yang, H. R. Li, and L. F. He: Beijing Biomed. Eng. 35 (2016) 26. https://doi.org/10.3969/ j.issn.1002-3208.2016.01.05

20 F. Miao, R. Z. Zhao, L. L. Jia, and X. L. Wang: Appl. Sci. 10 (2020) 3735. https://doi.org/10.3390/app10113735

21 Y. X. Lin, B. W. K. Ling, N. Xu, R. W. K. Lam, and C. Y. F. Ho: Biomed. Signal Process. Control 62 (2020) 102131. https://doi.org/10.1016/j.bspc.2020.102131

22 D. Asrina, T. E. Putra, and H. Husaini: Int. J. Integrated Eng. 12 (2020) 191. https://doi.org/10.30880/ ijie.2020.12.05.023 


\section{About the Authors}

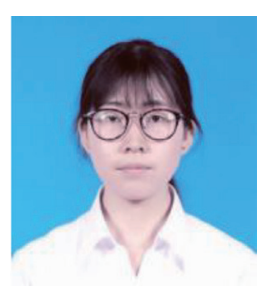

Qian-Hao Cheng received his B.S. degree in remote sensing science and technology from Beijing University of Civil Engineering and Architecture, China, in 2019. She is currently a graduate student majoring in photogrammetry and remote sensing at Beijing University of Civil Engineering and Architecture. Her research interest is bridge dynamic deflection signal denoising based on GBSAR.

(2108521519015@stu.bucea.edu.cn)

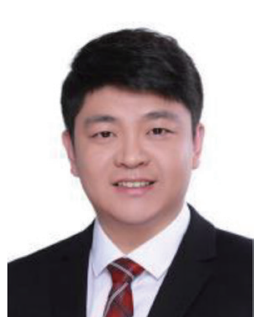

Qiang Chen received his B.S. degree in mapping engineering from China University of Mining and Technology, Beijing, China, in 2009, and his M.S. and $\mathrm{Ph} . \mathrm{D}$. degrees in cartography and geographic information system from Beijing Normal University, Beijing, China, in 2012 and 2017, respectively. His research interests include high-resolution remote sensing image analysis, urban refined information management, urban thermal space remote sensing analysis, urban natural disaster emergency response, GIS, and smart city management.

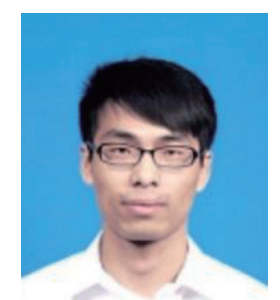

Hui Wang received his B.S. degree in remote sensing science and technology from Beijing University of Civil Engineering and Architecture, China, in 2018. He is currently a graduate student majoring in photogrammetry and remote sensing at Beijing University of Civil Engineering and Architecture. His research interest is bridge dynamic deflection signal denoising based on GBSAR.(2108160218005@stu.bucea.edu.cn)

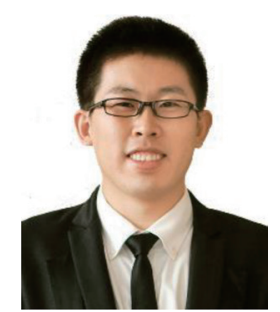

Xiang-Lei Liu received his B.S. and M.S. degrees in geographic information systems from Shandong University of Science and Technology in 2005 and 2008, respectively. He received his Ph.D. degree in photogrammetry and remote sensing from Tongji University in 2012. He is currently a professor of Beijing University of Civil Engineering and Architecture. His research interests are deformation monitoring based on GBSAR and high-speed videogrammetric measurement. 